Interpellation du genre et sujets mélancoliques. Butler lectrice d'Althusser

Interpellazione di genere e soggetti melanconici. Butler lettrice di Althusser

Jessica Borotto

(2) OpenEdition

Journals

Édition électronique

URL : http://journals.openedition.org/grm/715

DOI : $10.4000 / \mathrm{grm} .715$

ISSN : 1775-3902

Éditeur

Groupe de Recherches Matérialistes

Référence électronique

Jessica Borotto, «Interpellation du genre et sujets mélancoliques. Butler lectrice d'Althusser », Cahiers du GRM [En ligne], 8 | 2015, mis en ligne le 30 décembre 2015, consulté le 19 avril 2019. URL : http:// journals.openedition.org/grm/715; DOI : 10.4000/grm.715

Ce document a été généré automatiquement le 19 avril 2019

(c) GRM - Association 


\title{
Interpellation du genre et sujets mélancoliques. Butler lectrice d'Althusser
}

\author{
Interpellazione di genere e soggetti melanconici. Butler lettrice di Althusser
}

\author{
Jessica Borotto
}

\section{Introduction}

1 Cet article vise à explorer la manière dont Judith Butler remobilise la thèse althussérienne de l'interpellation en l'intégrant à une théorie de l'attachement passionné, à l'aide d'une compréhension psychanalytique de la formation du sujet. L'ouvrage qu'elle consacre à cette question est $L a$ Vie psychique du pouvoir ${ }^{1}$. Son travail nous intéresse en tant que tentative de rendre possible une relation critique à l'interpellation. Nous essaierons de comprendre comment l'articulation de l'interpellation althussérienne à cette figure de la psychanalyse qu'est la mélancolie correspond à cette tentative ${ }^{2}$. Notre but est de montrer la manière dont Butler a réinvesti l'interpellation althussérienne dans sa propre théorie, en insistant sur les éléments de sa lecture qui nous paraissent enrichir la problématique althussérienne. Nous suggérons que la position de Butler, consistant à se concentrer sur la dimension psychique du pouvoir, représente moins une alternative à l'analyse du fonctionnement des appareils idéologiques d'État qu'un complément permettant de complexifier le problème de la relation du sujet au pouvoir ${ }^{3}$.

2 Nous examinerons, dans un premier temps, l'analyse plus générale que Butler propose sur le fonctionnement psychique du pouvoir. Celle-ci interroge plusieurs auteurs dont certains nous aideront à tisser des liens précis entre l'interpellation chez Althusser et la subjectivation chez Butler. Après avoir abordé la question de l'adhésion subjective au pouvoir, nous préciserons en quoi la matérialité et l'ambiguïté des mécanismes de subjectivation deviennent, chez Butler, les sources d'une relation critique à l'interpellation. Cela nous permettra d'insister sur les éléments de la théorie althussérienne de l'interpellation et de l'idéologie qui servent de points de départ pour 
l'élaboration butlérienne d'une compréhension critique des formes de subjectivation. Il nous paraît utile à ce propos de les chercher non seulement dans le texte d'Althusser et dans les paragraphes que Butler lui consacre, mais également là où elle cherche à comprendre la mélancolie du genre comme l'effet d'une certaine interpellation et le deuil comme une réponse critique.

\section{Interpellation et subjectivation}

Dans La Vie psychique du pouvoir, Butler, enquêtant sur la problématique de l'assujettissement, se situe au croisement de différentes traditions (principalement les traditions hégélienne, foucaldienne et psychanalytique). L'articulation de certains éléments hérités de ces trois traditions et de la théorie nietzschéenne de la mauvaise conscience lui permet d'explorer, en l'enrichissant, le problème de l'ambivalence paradoxale de l'interpellation et de l'action du pouvoir. Une telle ambivalence s'explique par le fait que l'action du pouvoir non seulement obtient l'effet de subordination du sujet comme si elle agissait depuis l'extérieur de celui-ci, mais elle produit un tel effet aussi en opérant depuis son intérieur. En s'inscrivant ainsi dans une tradition initiée par Foucault, Butler conçoit l'assujettissement comme une dépendance envers un discours qui d'une part détermine les limites de l'existence sociale et de l'action du sujet et d'autre part les rend possibles ${ }^{4}$. Althusser pourrait lui aussi être situé à l'origine de cette tradition, dans la mesure où sa théorie de l'interpellation constitue une composante de l'horizon théorique dans lequel Foucault a élaboré sa conception de la production discursive du sujet.

4 Le texte d'Althusser sur lequel Butler concentre son analyse est « Idéologie et Appareils idéologiques d'État ", dans lequel apparaît la formule très connue et emblématique de l'interpellation: «Hé, vous, là-bas! $»^{5}$, le cri du policier qui fait se retourner l'individu. Dans cet ouvrage, la théorie de l'interpellation est un élément de la réflexion sur le rôle de l'idéologie dans la reproduction sociale. L'idée générale est que pour garantir la reproduction des rapports sociaux de production, l'idéologie interpelle les individus en sujets, autrement dit elle a pour fonction de les assujettir et de les subjectiver. Comme Butler le signale elle-même, dans "Idéologie et Appareils idéologiques d'État », la subordination du sujet interpellé se fait aussi par le langage, en ce qu'elle est l'effet produit par la voix qui appelle l'individu et qui lui attribue un nom. Le langage qui œuvre dans l'interpellation montrerait donc un pouvoir performatif qui aurait préparé les fondements de la notion foucaldienne de discours ${ }^{6}$.

Que la théorie de l'idéologie chez Althusser et la conception de la norme chez Foucault présentent de nombreux points en commun, c'est une position que Pierre Macherey défend dans Le Sujet des normes pour poser les bases de sa vision de la société des normes comme société idéologique. Dans ce livre, il met l'accent sur le caractère performatif ou productif de l'interpellation ainsi que sur sa matérialité : Althusser cherche à matérialiser l'idéologie, ce qui lui permet d'en développer une conception positive « en tant qu'agent effectif du processus de la reproduction sociale $»^{7}$ et de la production des sujets. Ces caractéristiques rapprochent l'idéologie de la norme chez Foucault, les deux concepts étant également voués à expliquer les processus de subjectivation comme des mécanismes de production et de subordination des sujets qui interviennent au niveau des pratiques et des comportements. Leur action est immanente aux effets qu'ils produisent : 
«Si l'idéologie (ou la norme) transforme, ce n'est pas en pénétrant progressivement un terrain préexistant à son action $»^{8}$, puisqu'elle produit d'emblée le terrain en question.

Guillaume Le Blanc, dans l'article «Etre assujetti : Althusser, Foucault, Butler ", tente de situer l'analyse menée par Butler sur la vie subjective du pouvoir vis-à-vis de Foucault et d'Althusser, en relevant lui aussi les éléments d'affinité qui rapprochent l'institution disciplinaire des appareils idéologiques d'État. La possibilité de faire converger les perspectives des deux auteurs ressort dans cette analyse - comme dans celle de Macherey - du fait que, d'une part, le pouvoir tel qu'ils le théorisent présente un caractère productif, et que, d'autre part, sa matérialité est indissociable de la dimension de l'idéal ou de l'idéologique. C'est par le biais de ces deux éléments que Butler se positionne en continuité avec Foucault et Althusser, bien qu'elle déplace son attention de la dimension objective de l'action du pouvoir à la dimension subjective. Le Blanc met ainsi en évidence que le fait de prolonger les théories d'Althusser et de Foucault dans une réflexion sur le fonctionnement de la vie psychique du pouvoir n'implique pas pour Butler, comme on pourrait le croire, la mise en question du caractère productif de celui-ci. Cela lui permet au contraire de complexifier l'analyse de la productivité de la relation au pouvoir dans la mesure où le sujet n'apparaît pas simplement comme sa cible, ni comme un produit fini, mais comme un processus qui s'effectue à travers sa relation au pouvoir. En d'autres termes, le travail de Butler montre comment les relations de pouvoir se réalisent en même temps que le sujet se produit.

\section{Sujets vulnérables et dépendance originaire}

7 La question qui est au cœur de La Vie psychique du pouvoir est la compréhension de la dépendance originaire sur le fond de laquelle le sujet émerge. L'insistance sur cette dimension et l'enquête plus précise sur les éléments qui rendent l'individu susceptible de se retourner (ou non) vers la voix qui l'interpelle visent à appréhender la vulnérabilité du sujet vis-à-vis du pouvoir qui l'assujettit ${ }^{10}$. Ces éléments témoignent de l'action du pouvoir sur et dans le sujet au cours de sa constitution, et leur repérage revient à déterminer ce sur quoi le sujet devrait avoir une prise pour se dé-subjectiver en vue d'autres formes de subjectivation. En outre, puisque prendre le risque de s'opposer à la loi implique le courage et même le désir de défaire le soi, il nous semble qu'explorer la dimension psychique du pouvoir est pertinent afin de comprendre comment ce désir de se défaire de soi pourrait s'engendrer et se développer.

8 Judith Butler affirme que l'aptitude à répondre à l'interpellation repose sur une complicité préalable avec la loi qui ne peut pas être expliquée seulement par l'appel puisqu'elle opère à l'intérieur du devenir sujet. De quoi s'agit-il dans cette complicité ? Comment l'expliquer? Althusser rend compte de l'adhésion subjective au pouvoir qui interpelle, lorsqu'il prête attention au retournement physique du sujet vers la loi, et qu'il le motive par le triple mécanisme de croyance, de doute et de certitude : l'individu "se retourne, croyant-soupçonnant-sachant qu'il s'agit de lui $»^{11}$. Avant le sujet, il y a l'individu qui devient sujet à la suite de l'interpellation: "Toute idéologie a pour fonction (qui la définit) de "constituer" des individus concrets en sujets $»^{12}$. Or, nous pourrions nous demander si c'est bien dans cette figure de l'individu proposée par Althusser que nous devrions chercher l'aptitude au consentement. Bien que cela soit possible, Althusser n'en dit pas beaucoup plus. La seule explication qu'il fournit à propos de l'individu concerne son caractère abstrait: l'individu qui n'est pas encore sujet est ontologiquement 
indéterminé. Cette figure est utile pour Althusser, car, afin d'expliquer le devenir sujet, il a besoin de se référer à quelque chose qui ne serait pas encore sujet et qui néanmoins constituerait un point de départ à partir duquel le sujet peut advenir à l'être. Pourtant, il conçoit l'individu comme une abstraction : tout individu concret, écrit-il, est toujours déjà sujet :

Comme l'idéologie est éternelle, nous devons maintenant supprimer la forme de la temporalité dans laquelle nous avons représenté le fonctionnement de l'idéologie et dire : l'idéologie à toujours-déjà interpellé les individus en sujets, ce qui revient à préciser que les individus sont toujours-déjà interpellés par l'idéologie en sujets; ce qui nous conduit nécessairement à une dernière proposition: les individus sont toujours-déjà des sujets. Donc les individus sont « abstraits » par rapport aux sujets qu'ils sont toujours déjà ${ }^{13}$.

Si, comme Butler l'affirme, «le sujet est pour l'individu la circonstance linguistique qui lui permet d'acquérir et de reproduire l'intelligibilité, la condition linguistique de son existence et de son action ${ }^{14}$, alors l'individu ne peut exister concrètement sans le sujet, sans être toujours déjà sujet. Il nous semble ainsi important de relever qu'il n'est pas possible chez Butler, comme chez Althusser, d'établir une entité qui existerait avant le sujet : l'introjection des relations externes de pouvoir, leur incorporation, n'a pas lieu à partir d'une entité nue ou brute. Il n'est pas question, pour ces auteurs, d'établir un moment du processus de subjectivation comme moment originaire constituant la dimension interne et subjective, discernable des éléments externes et objectifs, puisque, au contraire, cette séparation n'est que le produit de l'assujettissement. Rien dans leurs réflexions ne suggère la possibilité d'identifier quelque chose comme le noyau originaire et authentique du sujet et, de plus, il nous semble que cette question ne les intéresse pas. Il est plutôt question de rendre intelligible le processus d'introjection à travers des figures qui restent ontologiquement indéterminées et intégrées dans le processus de subjectivation, voire dans le circuit que Butler définit comme circuit de la conscience. Contrairement à ce que Macherey semble proposer dans son article «Judith Butler et la théorie althussérienne de l'assujettissement ", ce qui préoccupe Butler n'est pas de trouver l'entité qui serait affectée par un désir de loi avant de devenir sujet, ce qui amènerait effectivement à postuler un sujet avant le sujet ${ }^{15}$, mais de déceler l'affection même qui, comprise comme opération de pouvoir, précède et engendre le sujet. Puisque cette opération laisse des traces dans le versant psychique du sujet, il est possible de la comprendre en analysant ces traces comme des effets de la sédimentation du pouvoir, sans avoir besoin de chercher une entité subjective qui en serait affectée avant le sujet.

Dès son introduction, Butler fait l'hypothèse que l'interpellation du sujet présuppose que la formation de la conscience ait déjà eu lieu. En effet, elle conçoit celle-ci non pas comme un attribut ou une partie du sujet, mais bien plutôt comme un élément spécifique du pouvoir, comme une opération psychique - ou mieux qui devient psychique ${ }^{16}$ - véhiculant une norme ${ }^{17}$.

11 La conscience ainsi conçue ne suppose pas une réflexivité préalable, au contraire, elle la constitue : puisqu'elle intervient à l'origine du processus de subjectivation, elle n'est pas encore une conscience claire et réfléchissante - cette dernière étant le produit de la subjectivation -, mais elle est l'acte inaugural qui établit le commencement du mouvement réfléchissant ${ }^{18}$.

12 Cette conscience, opération du pouvoir qui devient psychique, consiste en une sorte de début de retournement, qui établit le sujet comme réfléchissant et réflexif. Le sujet devient ainsi l'objet de sa propre réflexion, comme dans l'acte qui initie le tour à 180 
degrés de l'individu hélé dans la rue: «Hé, vous là-bas!». Paradoxalement, c'est en devenant objet pour soi-même que le sujet devient sujet ${ }^{19}$. Si le sujet peut devenir objet pour soi, objet de sa propre réflexivité, c'est seulement dans un langage qui ne lui appartient pas exclusivement, mais qui signe la marque de l'autre dans l'origine du soi. Chez Butler, le sujet ne trouve les signes de sa propre existence qu'en dehors de lui, dans des catégories et des termes d'autrui : c'est seulement dans ces termes et dans ces catégories qu'il peut se penser. Du fait que le sujet ne peut émerger que dans et par le langage, que son existence est confirmée par ce langage, les relations qui le lient aux autres et ces autres qui laissent leur trace en lui, lui préexistent toujours.

Une deuxième idée parcourt toute l'analyse que Butler déploie dans son livre: le mouvement réflexif de la conscience est régi par un besoin d'autopunition et par un sentiment de culpabilité. Butler consacre le chapitre "Circuits de la mauvaise conscience $»^{20}$ à Nietzsche et Freud pour montrer que chez l'un et l'autre la sphère interne du sujet est l'effet d'un interdit. Dans la Généalogie de la morale, Nietzsche affirme que le créditeur inflige une punition au débiteur lorsque celui-ci se révèle incapable de payer sa dette. Le châtiment engendrerait le sentiment de culpabilité ou la mauvaise conscience chez le débiteur, ce qui l'amènerait à intérioriser la punition et à créer l'âme, c'est-à-dire l'intériorité. ${ }^{21}$ Toutefois, pour Butler, la conscience (ou l'âme) et son sentiment de culpabilité ne peuvent se produire par un mouvement d'intériorisation, car intérieur et extérieur se forment en même temps ${ }^{22}$. L'interdit dont elle parle est ainsi une sorte de punition, qui ne peut pas être ontologiquement située, mais seulement rétrospectivement citée. Cette punition retourne les pulsions ${ }^{23}$ et les désirs, originairement orientés vers les objets du monde (ni intérieur ni extérieur), contre euxmêmes. Un tel retour produit une "habitude psychique d'autopunition $»^{24}$ qui se fait conscience (et conscience coupable), et la distingue du monde extérieur. L'inflexion du désir vers le soi dédouble ce dernier et produit la réflexivité : ce mouvement susciterait un autre désir qui est celui du circuit lui-même, c'est-à-dire le désir de l'opération du pouvoir - la conscience. Le mouvement d'autopunition semble ainsi correspondre à la réflexion de la conscience : si, d'une part, il pose des limites aux possibilités d'action du sujet, d'autre part, en en déterminant les confins, il rend celui-ci susceptible d'assumer une identité et d'être reconnu. Il s'ensuit que le désir de réflexivité implique aussi un désir d'assujettissement que Butler désigne par la notion d'attachement passionné au pouvoir ${ }^{25}$.

14 Nous pourrions le dire autrement: si le pouvoir est ambivalent, c'est-à-dire s'il ne se limite pas à régler une matière qui lui préexiste, mais s'il règle ce qu'il produit - le sujet -, alors l'existence du sujet dépend de ce pouvoir qui l'assujettit. C'est pourquoi nous sommes autorisés à affirmer que le sujet est attaché au pouvoir dans la mesure où il est attaché à son existence. Cet attachement est supposé préexister au sujet, car c'est précisément cela qui l'induit à réagir à l'interpellation au commencement de sa formation, de telle sorte que sa réponse lui permette de devenir un bon sujet - ou un sujet normal. Dans cette perspective l'interpellation aurait besoin d'un attachement passionné, comme condition préalable à son efficacité.

Bien qu'Althusser ne mentionne que fugacement la culpabilité qui peut être à l'origine du retournement du sujet, en la désignant plutôt comme un caractère non central ${ }^{26}$, Butler prend l'initiative d'attribuer à l'individu idéologique une culpabilité motrice du retournement. Comme Butler l'explicite ${ }^{27}$, ce qui motive son interprétation est, d'une part, le fait qu'Althusser se sert de la métaphore de l'appel nominatif de Dieu dans 
l'exemple de l'idéologie religieuse pour expliquer le fonctionnement de l'interpellation, et d'autre part, que la notion de conscience ${ }^{28}$ telle qu'Althusser la mobilise semble renforcer, d'après Butler, la dimension théologique de l'opération du pouvoir. Établir un fond de culpabilité sur lequel l'interpellé énoncerait sa réponse permet à Butler d'expliquer pourquoi le sujet idéologique réagit comme s'il n'y avait d'autre possibilité que de répondre, comme si une contrainte le mouvait par nécessité. Seul un moteur interne pourrait produire un tel effet : c'est uniquement parce que le sujet perçoit l'appel comme venant de son intimité qu'il lui est (presque) impossible de s'y soustraire.

Butler propose une lecture symptômale de la théorie de l'interpellation en l'intégrant à la notion de culpabilité et à la conception psychanalytique de l'attachement passionné, afin de la pousser à dépasser ce qu'elle considère être ses limites. Une lecture symptômale d'un texte, selon la définition qu'Althusser en donne dans Lire "Le Capital " ${ }^{29}$, à propos de sa propre lecture du Capital de Marx, doit permettre de rendre visibles les éléments implicites d'une théorie, dont elle ne peut pas rendre compte, les éléments théoriques non développés et qui demeurent impensés. L'un des symptômes de la théorie de l'interpellation d'Althusser que Butler relève est l'exemple de l'appel nominatif divin, par lequel il attribue, sans le dire, une sorte d'absoluité à l'autorité qu'il cherche à comprendre et critiquer. En employant la métaphore de l'interpellation religieuse, Althusser ferait de la performativité religieuse le paradigme de l'interpellation idéologique et confèrerait ainsi à l'autorité de la voix interpellante en général un pouvoir divin, c'est-à-dire impossible à refuser. Ce faisant, selon l'analyse de Butler, Althusser rejoue le mécanisme de l'interpellation de l'autorité divine et limite à l'avance toute possibilité pour le sujet interpellé d'assumer une posture critique ${ }^{30}$.

Quoique pertinente, cette dernière critique nous parait problématique dans l'ensemble de la démarche de Butler. Fabio Bruschi, dans « Appareils et sujets chez Althusser », montre que Butler risque de réintroduire une dimension théologique à travers la notion de culpabilité, là où elle voulait justement s'en débarrasser en critiquant l'exemple de l'interpellation religieuse ${ }^{31}$. En effet, si elle peut attribuer le sentiment de culpabilité au sujet idéologique c'est précisément en raison du cadre religieux au sein duquel ce dernier émerge dans les exemples d'Althusser. Pourquoi alors le liquider? Seule son exigence de prendre ses distances d'avec la représentation du pouvoir interpellant comme sujet souverain explique la critique butlérienne de la métaphore divine chez Althusser. Une telle image s'oppose non seulement à son analyse du fonctionnement du pouvoir et de la formation des sujets, mais aussi à la manière dont elle cherche à réélaborer la théorie althussérienne.

18 L'intérêt que Butler accorde à Althusser traduit l'objectif de mettre en lumière des éléments de sa théorie qui ouvrent à des formes de résistance possible à l'interpellation. Autrement dit, si d'une part, en proposant une lecture symptômale, Butler cherche à faire émerger les éléments de la théorie de l'interpellation qui limiteraient à son avis la compréhension critique de l'assujettissement, d'autre part c'est en accentuant et en remobilisant d'autres points de la théorie althussérienne elle-même qu'elle cherche à poser les bases pour une telle compréhension. Une critique de l'assujettissement exige de prendre en compte deux éléments en particulier: le premier est l'ambivalence qui caractérise les mécanismes de la subjectivation, et qui se répercute sur la dimension psychique du sujet. Ce premier élément peut être relevé par Butler dans les textes d'Althusser et réélaboré à partir de là. Le deuxième est le fait que la formation du sujet advient aussi par un mécanisme de forclusion qui produit un domaine d'abjection que le 
sujet doit répudier pour subsister : pour rendre compte de ce deuxième élément Butler recourt à la psychanalyse.

\section{Pouvoir vulnérable : ambivalence de la maîtrise et de la reproduction des savoir-faire}

Butler identifie l'expression de l'ambivalence du pouvoir et de la subjectivation chez Althusser avec l'idée que la maîtrise, la maitrise des « savoir-faire », est indissociable de la soumission. Autrement dit, disposer d'une compétence signifie aussi être assujetti, puisque c'est précisément par la reproduction des qualifications que les conditions de l'assujettissement sont remplies: le système de la production est toujours un système d'exploitation. Althusser considère donc que l'apprentissage qui accompagne l'acquisition d'une maitrise (surtout grâce à l'appareil idéologique scolaire) coïncide avec le processus à travers lequel les sujets se subjectivent en se situant à leur place dans un certain ordre social ${ }^{32}$. C'est en ce sens que la qualification correspond aussi, pour le sujet, à l'acquisition d'un statut identitaire. Pour que la maîtrise des savoir-faire et donc la place du sujet dans l'ordre social, ainsi que la croyance dans l'idéologie, soient instaurées et maintenues, les sujets doivent répéter des actions techniques ${ }^{33}$. Cette répétition, d'après Butler, ne correspond ni à une reproduction mécanique ni à une répétition volontariste dans la mesure où le sujet qui la réalise n'est jamais entièrement formé et autonome mais que, se façonnant au cours de cette répétition, il demeure assujetti. La maîtrise des savoir-faire conçue à la suite de la critique des normes de Butler, repose sur une incorporation des techniques, incorporation qui permet au sujet d'en faire une compétence propre ${ }^{34}$.

Butler suggère la possibilité de distinguer le pouvoir comme condition d'émergence du sujet, du pouvoir comme ce que le sujet exerce. Une telle distinction n'est possible que si l'on suppose que, dans la transmission du pouvoir aux sujets émergents qui en deviennent les vecteurs, la reproduction n'est pas mécanique, ni entièrement achevée. Au contraire, la propagation et la reproduction du pouvoir exposent celui-ci à des possibles transformations. Être porteur d'un savoir-faire implique alors pour le sujet la possibilité d'exercer un pouvoir qui, en raison de sa matérialité et de la contingence à laquelle la répétition l'expose, peut différer de celui qui l'a engendré et ouvrir ainsi un écart entre la répétition du savoir-faire et la reproduction de l'assujettissement ${ }^{35}$. Le passage d'une modalité à l'autre n'efface pas l'ambiguïté constitutive de tout pouvoir, mais au contraire la perpétue. Autrement dit, le sujet en tant qu'il exerce du pouvoir se fait vecteur et instrument de son propre assujettissement en limitant toute critique de la loi, critique qui fragiliserait ses conditions d'existence et d'action; néanmoins, les possibilités de son existence excèdent les déterminations de la loi en raison des discontinuités possibles entre une répétition et l'autre.

Pour notre propos, il nous semble important de rappeler que, chez Althusser, d'un côté, l'acte de se retourner nécessite une croyance (comprise dans le triple mouvement psychique du croire, du douter et du savoir) et les actions sont consciencieusement accomplies par un sujet qui croit ; de l'autre côté, c'est la répétition d'une performance qui rend possible l'adhésion à une croyance (à l'idéologie) : la croyance se forme dans la répétition des opérations ${ }^{36}$, ce qui nous induit à affirmer que le sujet peut croire être le sujet qu'il est grâce à la répétition rituelle des savoir-faire qui le constituent ${ }^{37}$, ou (comme dirait Butler) grâce à l'incorporation des normes et techniques corporelles. 

conséquence du rapport entre croyance et rituel, qui est la suivante : si le fait d'avoir sapé le dualisme ontologique, qui séparait matérialité et idéalité, nous oblige à constater que le point de vue critique ne peut se fonder sur une dimension idéologique, dimension qui serait donnée avant les pratiques et qui constituerait un lieu de la pensée à l'abri de la matérialité du pouvoir, le sujet peut néanmoins adopter un rapport critique à sa propre constitution précisément en raison de la matérialité de l'idéologie. Une telle matérialité, selon Butler, impose que l'idéologie (la norme chez Butler) soit répétée, réitérée, reproduite, pour s'affirmer, ce qui l'expose à la contingence de l'action concrète: autrement dit, le pouvoir requiert sa reproduction matérielle pour subsister, et c'est dans cette exigence que se situe sa vulnérabilité vis-à-vis des sujets dont il a besoin pour se propager.

Le rituel a lieu dans la répétition et la répétition implique la discontinuité du matériel, le caractère irréductible de la matérialité au phénoménal. L'intervalle auquel une répétition se produit n'apparait pas, au sens strict ; il est pour ainsi dire, l'absence donatrice du phénoménal. Mais cette non-apparition ou cette absence ne constituent pas pour autant des «idéalités", car elles sont liées à cette donation comme à leur nécessité constitutive bien qu'absente ${ }^{40}$.

Il nous semble possible de trouver cette idée chez Althusser quand il insiste sur le besoin de l'idéologie d'assurer activement la reproduction des rapports de production ${ }^{41}$ et de fournir la garantie que «tout est bien ainsi » et que «tout ira bien ». Ce qui montre que l'ordre des choses instauré par l'idéologie ne va pas de soi selon une force nécessitante ou un modèle déterministe, mais nécessite l'intervention régulière (ou rituelle) de l'idéologie dans les pratiques des sujets ${ }^{42}$.

$\mathrm{Si}$, pour exister, le sujet est contraint à réitérer les normes par la répétition de gestes et actions, celles-ci ont tout autant besoin d'être réaffirmées par les sujets, ce qui les expose au risque d'être mal reproduites : «Il n'est de sujets que par et pour leur assujettissement »43. Nous avions déjà mis en évidence qu'une mauvaise reproduction de la norme pouvait 
menacer les conditions d'existence du sujet. À présent, nous insistons sur le fait qu'elle pourrait aussi offrir l'occasion de redéfinir les conditions sociales d'existence et de vie :

Un tel échec de l'interpellation peut très bien miner la capacité du sujet à «être » identique à soi ; elle peut également ouvrir la voie à un type d'être plus ouvert, voire plus éthique, un type d'être d'avenir ou pour l'avenir ${ }^{44}$.

\section{Mécanismes de forclusion et déni de la dépendance}

Pour compléter notre raisonnement avant de conclure sur la figure de la mélancolie, il nous faut explorer le mécanisme de forclusion. À cet égard nous reprenons la conception de la conscience formulée par Butler, en essayant de montrer comment le circuit qu'elle identifie laisse quelque chose d'extérieur à cette même conscience, quelque chose qui devient la condition inintelligible de l'émergence et de la reproduction du sujet. D'ici découle l'idée que la répétition consciencieuse des savoir-faire reproduit non seulement les sujets et leurs conditions matérielles d'existence, non seulement le champ de ce qui peut être thématisé dans le discours, mais aussi un domaine opaque qui relève de l'excès. Butler n'hésite pas à interpréter la conscience thématisée par Althusser comme une instance qui façonne le champ d'intelligibilité et qui permet donc de délimiter ce qui est pensable dans le langage (au sein d'une idéologie ou d'un ordre normatif donnés). Ainsi, l'action de la conscience se présente comme une opération restrictive et contraignante, qui marque les seuils du sujet et de sa capacité d'action avant même qu'il soit formé ${ }^{45}$.

Pour Butler, il n'est pas question de chercher une forclusion structurelle sur laquelle se fonderait l'échec de l'interpellation, puisque le domaine d'objets qu'elle embrasse est toujours contingent et historique. Il s'agit plutôt de montrer en quoi l'incapacité de l'interpellation à déterminer le champ entier du sujet sans failles est l'élément saillant qui rend son échec possible.

En ouverture de ce texte, nous avions déjà rencontré la figure de l'attachement originaire ou passionné à la loi. Nous pouvons maintenant mieux comprendre pourquoi Butler le théorise à la fois comme la condition de possibilité du venir à l'être du sujet, en ce qu'il opère en moteur interne du mouvement de réponse à l'interpellation, et comme ce qui reste partiellement opaque pour le sujet. La trace de l'autre dans l'identité du sujet, trace qui témoigne de la dépendance originaire de celui-ci, doit demeurer inintelligible pour que le sujet supposé auto-transparent puisse émerger en tant qu'autonome et rayonner dans toute sa clarté. Dès lors, l'existence du sujet et la persistance de son identité nécessitent non seulement des relations de pouvoir, dont l'émergence du sujet dépend, mais aussi une dénégation de cette dépendance. Paradoxalement la négation de la dépendance originaire, de l'attachement passionné à la loi, constitue aussi la condition de possibilité de sa réitération ${ }^{46}$ de la même manière que l'idéologie ne peut fonctionner que dans la dénégation de son caractère idéologique ${ }^{47}$. Butler s'intéresse aux mécanismes psychiques de forclusion en vue d'une politisation des sources psychiques susceptibles d'assumer un rôle critique. Ce qui est nié peut être politiquement investi seulement à la condition qu'il soit reconnu: seule la reconnaissance du caractère idéologique de l'idéologie témoigne d'un point de vue non idéologique; seule la reconnaissance de l'attachement passionné crée les conditions de possibilité pour s'en défaire. C'est pour répondre à cette exigence que Butler s'intéresse à la mélancolie du genre ${ }^{48}$ et au deuil. 


\section{Mélancolie du genre}

30 La relecture par Butler de l'ouvrage de Freud Le Moi et le ça $a^{49}$ en conserve l'idée que la mélancolie se trouve au cœur du processus d'identification. La mélancolie correspond à un deuil qui reste inachevé en raison de l'interdiction de reconnaitre la perte d'un objet. C'est à travers l'incorporation de cet objet perdu qu'elle produit des effets d'identification : elle le conserve en s'identifiant à lui ${ }^{50}$. Si cet objet persiste dans le moi comme une de ses identifications constitutives, d'après Butler il hante l'identité du sujet. Un tel mécanisme constitue à la fois la condition d'une acceptation partielle de la perte de l'objet dans le monde, car il permet de le conserver dans le moi, et ce qui mène le sujet à désavouer la perte, précisément parce que la seule manière de s'y confronter est l'identification à l'objet.

31 La mélancolie peut être expliquée par la figure du tour, qui évoque le demi-tour physique de l'individu interpellé en sujet idéologique et le demi-tour de la conscience qui se dédouble (et ainsi s'interpelle). Dans la mélancolie, en effet, le moi se produit comme objet de réflexion (en tant qu'objet d'amour ${ }^{51}$ ) en se substituant à la perte : c'est là que Butler identifie le moment d'inauguration du moi mélancolique ${ }^{52}$. Une telle opération a pour effet d'ériger les frontières entre le psychique et le social, les dimensions interne et externe de l'individu. Elle structure la vie psychique et ordonne, en la produisant, la topographie interne au sujet à travers l'incorporation d'un ordre normatif. Puisque ce monde interne relève de la structure sociale, il ne peut être subverti que par la transformation simultanée de celle-ci ${ }^{33}$.

Or, il nous semble évident que la mélancolie à laquelle Butler se réfère ne doit pas être comprise comme une pathologie qui affecte certains sujets incapables de s'adapter à un système normatif, mais bien plutôt comme une affection sociale qui concerne les sujets normés. Cette idée ne contredit pas Freud lorsqu'il reconnaît, dans Le Moi et le ça, que le processus d'identification mélancolique présente la même structure que les premières identifications. Celles-ci sont à la base de la formation du moi de tout individu et elles confèrent à son caractère des traits durable ${ }^{54}$. Le texte semble également suggérer que ces premières identifications inscrivent le moi dans un genre. En effet, Freud affirme que la première et plus importante identification de l'individu, à l'origine de l'idéal du moi, est l'identification au père. Il va de soi que lorsqu'il parle de l'identification au père il se réfère au sujet masculin, c'est pourquoi il précise dans une note en bas de page que " pour simplifier l'exposé $~_{55}$ il ne traitera que de l'identification au père ${ }^{56}$.

Des pages qui précèdent et qui suivent cette affirmation, il émerge que, bien que Freud ne le thématise pas de manière explicite, l'interdiction de l'inceste et l'acquisition d'un genre supposent, selon lui, l'interdiction de l'homosexualité et l'inscription à la norme hétérosexuelle. L'expression principale de celle-ci, comme Butler le souligne souvent, est la logique qui oppose le désir de l'objet et l'identification à l'objet comme contradictoires, ce qui engendre la terreur d'être ce qu'on désire (une femme si on est un homme, un homme si on est une femme) et de désirer ce à quoi on s'est identifié (une femme si on est une femme et un homme si on est un homme). En effet, Freud soutient que, dans sa phase orale, l'individu ne connait pas la distinction entre l'investissement érotique de l'objet et l'identification à l'objet ${ }^{57}$. Ensuite il indique que, tant que la connaissance de la différence des sexes n'est pas encore acquise, mère et père sont indistinctement les premiers objets érotiques $^{58}$. Ce qui suggère que l'identification au père et le désir de la mère, possibles 
seulement lorsque la différence des sexes est connue, présupposent la perte désavouée ${ }^{59}$ du père comme objet d'amour. C'est pourquoi Butler fait l'hypothèse que le complexe d'œEdipe peut avoir lieu seulement si l'interdiction de l'homosexualité est déjà intégrée ${ }^{60}$.

Un tel système normatif produit un domaine de figures abjectes auxquelles le désir ne peut pas être adressé. Celles-ci constituent ainsi des objets perdus, mais dont la perte doit être niée puisque sa reconnaissance supposerait en même temps la possibilité du désir. À l'interdit du deuil correspond ainsi la prohibition de reconnaitre certaines formes de vie et certains types de relations comme légitimes. C'est en ce sens que la mélancolie, chez Butler, se caractérise comme la perte désavouée des possibilités jamais vécues (puisqu'objets d'interdiction). De ce fait la perte de la perte de l'autre se révèle être aussi la perte du monde social dans lequel cette perte pourrait être reconnue, ce qui rend possible la reproduction des conditions sociales données.

Dans la mélancolie, la perte d'un autre ou d'un idéal est elle-même perdue pour la conscience. À son tour, le monde social dans lequel une telle perte est devenue possible est également perdu. Le mélancolique non seulement soustrait l'objet perdu à la conscience, mais il soustrait à la psyché une configuration du monde social $^{61}$.

La forclusion de ce qui ne doit pas être pleuré publiquement nourrit la violence interne de la conscience contre les abjections et la partie du moi qui les désire en consolidant l'effet d'identification qui à son tour renforce l'ordre social. Nous semblons ainsi retrouver, dans le processus d'identification mélancolique, la même dynamique qui opère dans la relation entre rituel et croyance : d'une part c'est le sujet croyant qui agit de façon à reproduire les conditions matérielles de la croyance, d'autre part la répétition rituelle produit, maintient et renforce la croyance. De ce point de vue, l'inintelligibilité et l'illégitimité du désir homosexuel sont le résultat d'une réitération des techniques corporelles et d'habitudes discursives ritualisées : elles sont le fruit d'une répétition des savoir-faire jusqu'à leur maîtrise, maîtrise qui coïncide avec l'assomption bien réussie des normes du féminin et du masculin.

\section{Autres sujets, autres tours}

Or, comme Michael Foessel l'exprime clairement dans «Malaise dans l'identification. La mélancolie du genre ", s'il s'agit pour Butler de penser la mélancolie au-delà des cadres cliniques, pour l'inscrire dans le contexte plus large de la société, alors la transformer en deuil implique une politisation de celui-ci. Ce qui d'une part suppose de substituer les normes sociales au "principe de réalité " $^{62}$ de Freud et d'autre part demande une élaboration du rapport à soi qui engage davantage à la mutation des normes sociales qui habitent les individus qu'à la guérison individuelle comme adéquation au système normatif dominant ${ }^{63}$.

Comme nous le verrons de manière plus détaillée dans les paragraphes qui suivent, la mélancolie contient une colère qu'elle tend à diriger contre le moi ${ }^{64}$. Si la colère est réorientée et resignifiée dans une dimension collective, elle peut être traduite en expression politique capable d'amener à la reconnaissance du deuil. Son investissement politique ne va pourtant pas de soi, mais nécessite un travail particulier sur le sujet et des formes d'opposition visant à agir sur l'ordre social, sur ce qu'Althusser définirait comme les appareils idéologiques d'État. 

un sujet particulier : le sujet homosexuel. Dans la mesure où l'hétérosexualité constitue l'idéal normatif et oblige à respecter le binarisme de genre, celui-ci est à son tour affecté par une mélancolie qui résulte des déplacements des normes du féminin et du masculin vers des sujets qui ne leur correspondent pas. Il s'agirait de l'affection d'un «mauvais sujet », d'un sujet «mal subjectivé » à cause de l'échec de son positionnement dans le langage, à cause d'une répétition des savoir-faire et d'une ritualisation des techniques corporelles qui n'auraient pas dû être de sa compétence. lorsque les pertes des possibilités interdites sont avouées et entrent dans le champ de l'intelligible : puisque la logique binaire et identitaire régit la mélancolie, sa réfutation coïncide avec la reconnaissance du deuil. Le deuil, correspondant à la mélancolie du genre à condition qu'il soit publiquement exprimé, rendrait ainsi viables la dissociation du désir de la répudiation et une relation entre identification et désir qui ne serait pas nécessairement contradictoire ${ }^{65}$. Butler insiste sur l'importance de la publicité du deuil dans Antigone's claim ${ }^{66}$ en analysant l'Antigone de Sophocle : cette figure met en scène le fait que c'est seulement lorsque la perte peut entrer dans le champ du discours et dans le langage, lorsque la mémoire du frère perdu obtient reconnaissance officielle, que le deuil peut être estimé accompli. en termes psychanalytiques des conséquences très précises. Comme Butler l'indique, Freud, dans Deuil et mélancolie, caractérise la mélancolie par un mouvement d'autopunition, car le sujet oriente contre soi la punition qui devait s'adresser à l'objet perdu. Une telle colère dépend de l'ambiguïté caractérisant l'amour (amour-haine) que le sujet éprouvait pour l'objet perdu. Étant donné que celui-ci est maintenu à travers son incorporation par le processus d'identification, toute colère qui aurait été dirigée contre lui, colère qui se renforce au moment de la perte de l'objet, est réorientée contre le moi ${ }^{67}$. Cela engendre un conflit entre une partie du moi et le sur-moi, conflit qui institue une réflexivité et une instance critique. Le sur-moi juge ainsi le moi à l'aune d'un idéal du moi, idéal qui est le produit des identifications avec l'objet perdu et idéalisé. Cet idéal a un caractère social ${ }^{68}$ dans la mesure où il est le produit des identifications qui se sont engendrées à partir d'une interdiction sociale de reconnaissance du deuil.

Reconnaître la perte permettrait au sujet d'arrêter d'adresser les pulsions de mort contre soi, pour les ré-adresser contre l'objet perdu et donc lui permettrait de continuer à rester en vie en mettant fin au processus d'autodestruction du moi ${ }^{69}$. Bien entendu, cela ne signifie pas adresser la colère à l'objet en tant qu'abjection et objet indésirable, puisque

Cahiers du GRM, 8 | 2015 
cela amènerait à renforcer la mélancolie et à orienter les pulsions de mort aussi contre le moi désirant. Il nous semble que quand Butler affirme la nécessité d'adresser les pulsions de mort à l'objet perdu, elle décrit le processus du deuil pour lequel extérioriser les pulsions de mort contre l'objet perdu signifierait en reconnaître la perte et donc sa légitimité en tant qu'objet désirable. En outre, cela permettrait de diriger ces pulsions aussi contre l'idéal du moi qui l'avait remplacé (c'est pourquoi la colère doit trouver un horizon collectif et politique d'expression et de signification). C'est précisément au sein de cette opération qu'il est possible, pour Butler, de transformer les pulsions de mort en source politique et critique : ceci exige d'extérioriser la colère de telle sorte que celle-ci, dirigée contre l'idéal du moi, serait en même temps dirigée contre l'ordre social qu'il représente dans la vie psychique.

La colère est l'expression des pulsions de mort qui, comme nous l'avons vu au tout début de l'article, se retournent contre elles-mêmes, par une opération de pouvoir qui est la conscience, en produisant ainsi la dimension interne du sujet. L'action de la conscience consiste à marquer les seuils du moi afin de le rendre disponible pour les identifications et les formes de reconnaissance sociale, mais, pour ce faire, elle dirige les pulsions de mort vers son intérieur : c'est en ce sens qu'elle a aussi un effet mortifère, qu'elle mortifie la vie. Ce à quoi Butler oppose le désir de vivre : celui-ci coïncide avec un désir de désubjectivation, avec un désir de déconstruction de soi. L'extériorisation de la colère produit une sorte de dé-confinement du sujet en rendant ainsi possible sa déconstruction en opposition à l'action de la conscience qui enfermait le moi dans ses frontières et à son opération d'autopunition qui orientait les pulsions contre le moi et en établissait les seuils. Butler peut ainsi rendre compte d'une dynamique psychique pouvant devenir source de subversion collective et dans laquelle le désir de vivre ne correspond pas au désir du moi ni au désir de maîtrise du moi, mais à un désir de défaire le moi. En effet, son analyse complexe de la mélancolie montre que c'est la maîtrise du moi qui oriente les pulsions de mort contre le moi, tandis que le désir de vie défait cette maîtrise en posant les bases pour un mode de devenir-sujet qui diffère de celui du sujet interpellé en sujet mélancolique. C'est pourquoi, en opposition à Lacan, elle refuse de faire d'Antigone la figure du désir de mort $^{70}$.

Si le moi contient l'agression contre le disparu, il s'ensuit que la ré-extériorisation de cette agression « dé-contient » le moi. Le désir de vivre n'est pas le désir de moi, mais un désir qui défait le moi dans le procès de son émergence. La " maîtrise » du moi est définie comme effet de pulsion de mort. La vie, au sens nietzschéen du terme, détruit cette maîtrise ; elle instaure un mode de devenir qui contredit par sa vivacité la stase et le statut défensif ${ }^{71}$.

Si le deuil permet au sujet de se défaire de l'attachement passionné au pouvoir qui l'avait exposé à l'interpellation en sujet mélancolique, c'est seulement par la reconnaissance de la trace de l'autre effacé par cet effet de forclusion. Une telle reconnaissance mettrait en même temps en évidence la dépendance aux relations sociales de pouvoir sur fond desquelles le sujet émerge. C'est seulement en renonçant à l'autonomie et à l'autotransparence, dont la croyance et la certitude n'étaient que le produit d'un déni ritualisé, que le sujet peut se défaire.

C'est là, pour Butler, l'exemple d'un autre tour - un tour qui répond à l'interpellation en la transformant et en transformant ainsi ses effets. Il y a là aussi une manière de reconnaître le caractère idéologique de l'idéologie et de détourner le pouvoir acquis par la maîtrise des savoir-faire et du bien parler contre lui-même. 
Toutefois la question reste ouverte. Butler affirme que le deuil doit être toujours opérant pour éviter la fixation du sujet dans des formes d'identification mélancolique. En effet, il ne peut jamais se libérer entièrement des relations de pouvoir; il peut uniquement les transformer, mais il en garde toujours l'ambivalence. Il s'agit alors de prendre en compte le fait que, en raison de l'ambivalence de tout pouvoir, la reconnaissance d'un deuil et d'un objet d'amour peut aussi devenir un outil de nouvelles formes de subjectivation.

Un autre vaste champ de questions s'ouvre si nous considérons qu'il y a des formes de subjectivation qui n'opèrent pas directement sur le genre, auxquelles l'analyse de la mélancolie du genre peut ou doit se confronter.

Nous pouvons néanmoins conclure ainsi : en remobilisant la théorie althussérienne de l'interpellation, Butler nous suggère que pour le sujet, il est possible de transformer la relation à ses conditions d'existence ainsi qu'à ses frontières, car vis-à-vis de la voix interpellante, « Hé vous, là bas ! », il existe toujours des réponses inédites.

\section{NOTES}

1. Judith Butler, La Vie psychique du pouvoir. L'assujettissement en théories, tr. fr. B. Matthieussent, Paris, Editions Léo Scheer, 2002.

2. Butler précise qu'elle ne vise pas à défendre une « approche empirique », ni à « rendre compte des positions psychanalytiques présentes sur le genre ", mais "seulement à suggérer quelques convergences productives entre la pensée de Freud sur le deuil et les pertes impossibles et le fait de vivre dans une culture qui a beaucoup de mal à accepter le deuil d'un attachement homosexuel » (ibid., p. 207).

3. Soulignons que, dans d'autres ouvrages, Butler s'intéresse également au versant objectif du pouvoir et de la formation des sujets. Elle développe des réflexions critiques sur les dispositifs du pouvoir d'Etat, juridiques, militaires ou médiatiques dans Ce qui fait une vie. Essai sur la violence, la guerre, le deuil (tr. fr. J. Marelli, Paris, La Découverte, 2010), et dans Vies précaires. Le pouvoir du deuil et de la violence après le 11 septembre 2001 (tr. fr. J. Rosanvallon et J. Vidal, Paris, Editions Amsterdam, 2005), où elle aborde la question de la distribution différentielle de la précarité de la vie et des cadres - frames - d'intelligibilité spécifiques à des contextes de guerre et de migration qui produisent les conditions et les limites de la reconnaissance des sujets et de la valeur de leur vie.

4. J. Butler, La Vie psychique du pouvoir. L'assujettissement en théories, op. cit., p. 22.

5. Louis Althusser, « Idéologies et appareils idéologiques d'Etat » in Sur la reproduction, Paris, PUF, 1995 p. 305.

6. J. Butler, La Vie psychique du pouvoir. L'assujettissement en théories, op. cit., p 26.

7. Pierre Macherey, Le Sujet des normes, Paris, Editions Amsterdam, 2014, p. 52.

8. Ibid., p. 93. Une partie du chapitre «Figures de l'assujettissement » dont nous avons tiré les citations est disponible en ligne : P. Macherey, Deux figures de l'interpellation : "Hé, vous, là-bas!» (Althusser) - «Tiens, un nègre!» (Fanon), mis en ligne le 15 février 2012 (consulté la dernière fois le 1 juin 2015) URL : http://philolarge.hypotheses.org/1201.

9. Guillaume Le Blanc, Etre assujetti: Althusser, Foucault, Butler, in «Actuel Marx », 2004/2 n³6, p. 45-62. 
10. J. Butler, La Vie psychique du pouvoir. L'assujettissement en théories, op. cit., p. 167-178.

11. L. Althusser, «Idéologies et appareils idéologiques d'Etat », op. cit., p. 306.

12. Ibid., p. 303 (c'est Althusser qui souligne).

13. Ibid., p. 306-307.

14. J. Butler, La Vie psychique du pouvoir. L'assujettissement en théories, op. cit., p. 34.

15. «Non seulement la voix qui lance l'appel auquel répond le geste de retournement est censée se faire obéir, et ceci peut-on dire "sans appel", mais elle procède d'une logique punitive qui ne fonctionnerait pas si elle ne s'appuyait, de la part de "celui" auquel elle s'applique, sur un sentiment ou une reconnaissance potentielle de culpabilité qui donne à la scène de l'interpellation son horizon d'attente. C'est pourquoi la production de sujet, telle que l'avère la déclaration d'obéissance une fois celle-ci obtenue, suppose comme préalable quelque chose comme une intention ou un désir d'obéir, inconscients bien sûr, dont le siège ne peut être qu'un "celui" ou un sujet préexistant à la production de sujet: il en résulte que, pour que s'effectue réellement cette production, il faut qu'il y ait déjà sujet, porteur de la pulsion de culpabilité qui incline à se soumettre à la voix qui appelle, en adoptant une posture d'obéissance qui ne correspond pas seulement au fonctionnement d'un automatisme parce qu'elle consiste aussi en l'accomplissement d'un désir ». (P. Macherey, "Judith Butler et la théorie althussérienne de l'assujettissement ", in P. Macherey, Le Sujet des normes, op.cit., p. 371). Ce texte est également disponible en ligne : P. Macherey, Judith Butler et la théorie althussérienne de l'assujettissement, mis en ligne le 18 février 2002 (consulté fois le 1 juin 2015), URL : http://stl.recherche.univ-lille3.fr/ seminaires/philosophie/macherey/macherey20082009/macherey18022009.html

16. Nous ajoutons cette précision: l'opération psychique du pouvoir entendue comme l'« inauguration tropologique du sujet » (J. Butler, La Vie psychique du pouvoir. L'assujettissement en théories, op.cit., p. 24), doit être conçue comme une opération qui devient psychique. Lorsque Butler soutient que la conscience (qui n'est pas encore une conscience réfléchissante, mais l'acte initiateur de la réflexion) est supposée par l'interpellation et qu'elle la désigne comme une «opération psychique» (Ibid., p. 27), elle nous induit à penser que la dimension psychique précède l'interpellation inaugurale. Toutefois il nous semble clair que, pour elle, la dimension psychique du pouvoir commence à se distinguer en même temps que le pouvoir interpelle.

17. Ibid., p. 27.

18. Ibid., p. 176-177.

19. Nous pouvons formuler des énoncés comme celui qui dit que le «sujet » devient "sujet » seulement rétrospectivement puisque justement le premier terme dont il est question n'est pas encore sujet!

20. Ibid., p. 107-133.

21. Friedrich Nietzsche, "La "faute", la "mauvaise conscience" et ce qui leur ressemble» in La généalogie de la morale, trad. Fr. I. Hildenbrand et J. Gratien, Paris, Gallimard, 1971.

22. Butler insiste sur le caractère circulaire de la relation entre la punition extérieure et l'autopunition, entre la dette et la production de l'âme. Si, d'une part, le débiteur est celui qui ne sait pas tenir une promesse ni persévérer dans sa volonté, et si une telle capacité nécessite une âme, d'autre part, celle-ci est produite par la punition. En effet, la mauvaise conscience est l'autoapplication de la culpabilité et induit l'autopunition. Celle-ci coïncide avec l'intériorisation de l'instinct (ou de la pulsion) qui produit l'âme. L'âme, pour se former, a donc besoin de l'intériorisation de la punition qui n'a lieu que lorsque le débiteur ne tient pas sa promesse, en brisant ainsi le contrat avec le créditeur. Toutefois, pour se justifier, la punition présuppose le modèle de la volonté souveraine, entière et continue qui maintient sa promesse (J. Butler, La Vie psychique du pouvoir. L'assujettissement en théories, op. cit., p. 121-123).

23. Nietzsche parle plutôt d'instinct, mais après avoir articulé la théorie de la mauvaise conscience chez Nietzsche à celle de la formation du moi chez Freud, Butler privilégie le lexique psychanalytique. Les pulsions en question comprennent aussi des pulsions de mort qui, dans la 
mélancolie, sont réorientées contre le moi et s'expriment en forme d'autopunition et de culpabilisation.

24. Ibid., p. 50.

25. Ibid., p. 47-48.

26. L. Althusser, «Idéologies et appareils idéologiques d'Etat », op. cit., p. 305.

27. J. Butler, La Vie psychique du pouvoir. L'assujettissement en théories, op. cit., p. 169, 170.

28. Ibid., p. 181.

29. L. Althusser, «Du Capital à la philosophie de Marx » dans AA. VV., Lire «Le Capital », Paris, Editions Maspero, 1973, p. 36.

30. J. Butler, La Vie psychique du pouvoir. L'assujettissement en théories, op. cit., p. 169-171.

31. Fabio Bruschi, «Appareils et sujets chez Althusser ", Cahiers du GRM, $4 \mid 2013$, mis en ligne le 12 décembre 2013, consulté le 10 juin 2015. URL : http://grm.revues.org/357, p. 3.

32. L. Althusser, «Idéologies et appareils idéologiques d'Etat », op. cit., p. 272-274 et p. 290-291.

33. Nous pouvons retrouver cette idée de répétition dans la notion de rituel : les « pratiques sont réglées par des rituels » dans «l'existence matérielle d'un appareil idéologique » (ibid., p. 300-301).

34. J. Butler, La Vie psychique du pouvoir. L'assujettissement en théories, op. cit., p. 180-182.

35. Ibid., p. 38-39.

36. L. Althusser, «Idéologies et appareils idéologiques d'Etat », op. cit., p. 300-301.

37. Ibid., p. 240-242.

38. Ibid., p. 302.

39. Ibid., p. 299-302.

40. J. Butler, La Vie psychique du pouvoir. L'assujettissement en théories, op. cit., p. 192-193.

41. L. Althusser «Idéologies et appareils idéologiques d'Etat », op. cit., p. 239-240.

42. Ibid., p. 312.

43. Ibid., p. 311 (c'est Althusser qui souligne).

44. J. Butler, La Vie psychique du pouvoir. L'assujettissement en théories, op. cit., p. 198.

45. Ibid., p. 176- 177.

46. Ibid., p. 31.

47. L. Althusser, «Idéologies et appareils idéologiques d'Etat », op. cit., p. 306.

48. J. Butler, La Vie psychique du pouvoir. L'assujettissement en théories, op. cit., p. 199-285. Ce n'est pas alors la première fois que Butler élabore ses thèses sur la mélancolie de genre; elle avait déjà commencé à les développer à partir des relectures de la mélancolie chez Freud, Kristeva et Irigaray, dans "Freud et la mélancolie du genre», "Complexité de genre et limites de l'identification ", in Trouble dans le genre. Le féminisme et la subversion de l'identité, tr. fr. C. Kraus, Paris, La Découverte, 2006, p. 147-168. Elle a ensuite repris la figure de la mélancolie en relation au drag dans «La mélancolie et les limites de la performance", in Ces corps qui comptent. De la matérialité et des limites discursives du « sexe », tr. fr. C. Nordmann, Paris, Editions Amsterdam, 2009, p. 236-239.

49. Sigmund Freud, Le Moi et le ça, tr. fr., J. Laplanche, Paris, Editions Payot et Rivages, 2010.

50. Ibid., p. 70.

51. Ibid., p. 73.

52. J. Butler, La Vie psychique du pouvoir. L'assujettissement en théories, op. cit., p. 264.

53. Ibid., p. 245-253.

54. S. Freud, Le Moi et le ça, op. cit., p. 70.

55. Ibid., p. 75.

56. Ibid., p. 74-75.

57. Ibid., p. 70.

58. Ibid., p. 75.

59. Nous pouvons déduire que la perte de cet objet d'amour est désavouée du fait que son investissement érotique ne fait même pas l'objet d'une interdiction explicitement thématisée. 
60. J. Butler, La Vie psychique du pouvoir. L'assujettissement en théories, op. cit., p. 203.

61. Ibid., p. 264.

62. S. Freud, Le Moi et le ça, op. cit. p. 64.

63. Michaël Foessel, «Malaise dans l'indentification. La mélancolie du genre ", in Fabienne Brugère et Guillaume Le Blanc (éd.), Trouble dans le sujet, trouble dans les normes, Paris, PUF, 2009, p. 104.

64. Il nous semble qu'à partir de l'analyse que Butler fait de la mélancolie, nous pouvons soutenir que la colère est adressée non seulement au moi, au moi désirant l'objet perdu, ce qui produit des restrictions qui contiennent le moi dans une identité rigide (et qui peuvent l'amener jusqu'à la mort), mais aussi contre l'objet perdu qui, n'étant pas reconnu comme objet désiré, devient abjection: la mélancolie hétérosexuelle produit des effet de mépris et refus vis-à-vis des homosexuels qui expriment aussi un type de colère.

65. Elle prend en exemple le Names projects aids memorial quilt (J. Butler, La Vie psychique du pouvoir. L'assujettissement en théories, op. cit., p. 220).

66. J. Butler, Antigone's claim. Kinship between life and death, New York, Columbia University Press, 2000.

67. S. Freud, «Deuil et mélancolie», tr. fr. M. Bonaparte et A. Berman, in Euvres complètes. Psychanalyse, vol. XIII, Paris, PUF, 2005, p. 268-272.

68. S. Freud, Le Moi et le ça, op. cit. p. 84-86.

69. S. Freud, « Deuil et mélancolie », op. cit., p. 279.

70. Jacques Lacan, «L'Eclat d'Antigone », in Ethique de la psychanalyse. Séminaire VII, Paris, Edition du Seuil, 1986.

71. J. Butler, La Vie psychique du pouvoir. L'assujettissement en théories, op. cit., p. 280.

\section{RÉSUMÉS}

Cet article propose d'explorer la manière dont Judith Butler remobilise la thèse althussérienne de l'interpellation dans La Vie psychique du pouvoir . Nous essaierons de saisir les éléments qui nous paraissent enrichir la problématique althussérienne, suggérant que la compréhension de la dimension psychique du pouvoir qui ressort de sa lecture permet de complexifier la relation du sujet au pouvoir. Afin de tisser des liens précis entre l'interpellation chez Althusser et la subjectivation chez Butler, nous examinerons d'abord le rôle que Nietzsche et Foucault recouvrent dans la formulation de la théorie de la subjectivation chez Butler. Ensuite, il s'agit de comprendre en quoi la matérialité et l'ambiguïté des mécanismes de subjectivation, qui déjà caractérisent l'interpellation althussérienne, deviennent chez Butler les sources d'une relation critique aux conditions d'émergence du sujet. Enfin nous préciserons comment elle mobilise de telles sources pour élaborer cette figure de la psychanalyse qu'est la mélancolie et pour décrire sa transformation en deuil.

Questo articolo propone di approfondire la maniera in cui Judith Butler mobilizza la tesi dell'interpellazione di Althusser ne La vita psichica del potere. Nell'intento di suggerire che la sua comprensione della dimensione psichica del potere rende più complessa la relazione tra questo $\mathrm{e}$ il soggetto, cercheremo di cogliere gli elementi che ci sembrano arricchire le problematiche sollevate da Althusser. In primo luogo esamineremo il ruolo che Nietzsche et Foucault ricoprono nella formulazione della teoria della soggettivazione di Butler e metteremo in evidenza gli 
elementi sui quali tale teoria si situa in continuità con Althusser. In seguito cercheremo di comprendere in che misura Butler fa della materialità e dell'ambiguità dei meccanismi di soggettivazione, che già caratterizzano l'interpellazione di Althusser, delle risorse per una relazione critica alle condizioni di emergenza del soggetto. Infine chiariremo come essa mobilizza tali risorse nell'elaborare la figura psicanalitica della melanconia e nel descrivere la sua trasformazione in lutto.

\section{INDEX}

Index géographique : Europe

Index chronologique : XXe siècle

Thèmes : psychanalyse, marxisme, philosophie politique, matérialisme

Mots-clés : subjectivation, interpellation, genre, mélancolie, sujet, pouvoir, idéologie, normes, Butler Judith, Althusser

\section{AUTEUR}

\section{JESSICA BOROTTO}

Jessica Borotto est aspirante FRS-FNRS attachée à l'UR MAP de l'Université de Liège. Elle mène actuellement une thèse de doctorat intitulée : «Langage, corps, pouvoir. Apports de la théorie de la subjectivation de Judith Butler à l'étude des enjeux éthiques et politiques des savoirs biomédicaux contemporains » qui s'inscrit dans le projet de recherche GIGS (Gouvernementalité de l'information génomique et de la santé). 